\title{
The Space Debris Growth Model
}

\author{
Sijia Chen \\ School of Electric Power Engineering, North China Electric Power University, Baoding 071000, \\ China. \\ 348661849@qq.com
}

Keywords: space debris, Simulated Annealing, least square method.

\begin{abstract}
Now space debris poses a serious threat to humanities efforts at space exploration as well as the expanding uses for earth-orbiting satellites. This thesis is to predict the growth trend of space debris. We construct the Space Debris Growth Model. Based on statistics in past years, Least Squares and Simulated Annealing are used to obtain the parameters of the growth trend fitted curve. Then we obtain the changing tendency of space debris quantity along with the time. It is shown that the quantity and rising rate continually increase.
\end{abstract}

\section{Introduction}

Since 1957, the Soviet Union launched Sputnik satellite. Over the last few decades, the rate at which human activities in space have led to the creation of space debris has increased in a linear fashion. As the film $<$ Gravity $>$ presents a huge disaster caused by space debris, many space debris have posed a great threat to human space resource development activities. For example, the Russian satellite Kosmos-2251 and the USA satellite Iridium-33 collided on 10 February, 2009, which have a great impact on space environment. Herein, we show two pictures ${ }^{[1]}$ to illustrate the impact of space debris on the earth:
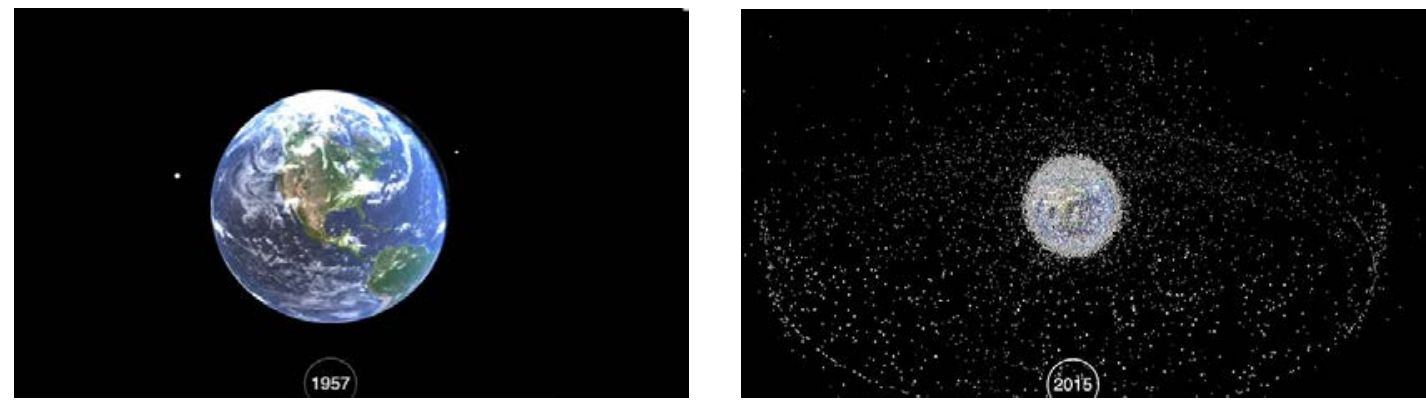

Figure 1.The earth surrounded by space debris in 1957 and 2015

\section{Model Justification}

As the space environment constantly deteriorates, many countries and institutes have been involved in clearing out the space debris. In order to clear up the space debris more efficiently, we should predict the growth tendency of the space debris with time going by, to make the time arrangement of clearing the space junk. Meanwhile, since the growth trend of the space debris will also decide the methods we will choose to clear, the scale of the clear work and so on, studying the growth trend of the space debris is an important step of our model.

The space debris can be divided into three categories according to their size: large debris $(>10 \mathrm{~cm})$, dangerous debris $(1 \mathrm{~cm} \sim 10 \mathrm{~cm})$ and small debris $(<1 \mathrm{~cm})^{[1]}$. The pieces of which size is $1 \mathrm{~cm} \sim 10 \mathrm{~cm}$, have very high speed and large quantity, consulting in they are not easy to observe. We called this kind of debris as dangerous debris, which is the key objectives in clearing out space debris because their serious destruction. While the large pieces $(>10 \mathrm{~cm})$ have larger volume ,we can track them to get their orbit parameters for spacecraft and make instructions to avoid collision crisis in time .Besides, though there are many little debris $(<1 \mathrm{~cm})$ in the space, we can ignore their damage since their volume is smaller. ${ }^{[1]}$ Based on this, we can only consider the impact of the dangerous 
debris to simplify our model. Considering that the number of the all kinds of debris is unknown, but the number of various types of space debris in the total number of space debris occupies a fixed proportion, so we research the tendency of the number of the total space debris and multiply by the fixed proportion to obtain the tendency of the number of the various types of debris.

\section{Model Formulation}

Using the historical data from website ${ }^{[2]}$, we draw the scattered point distribution of the space debris, and the results are as follows:

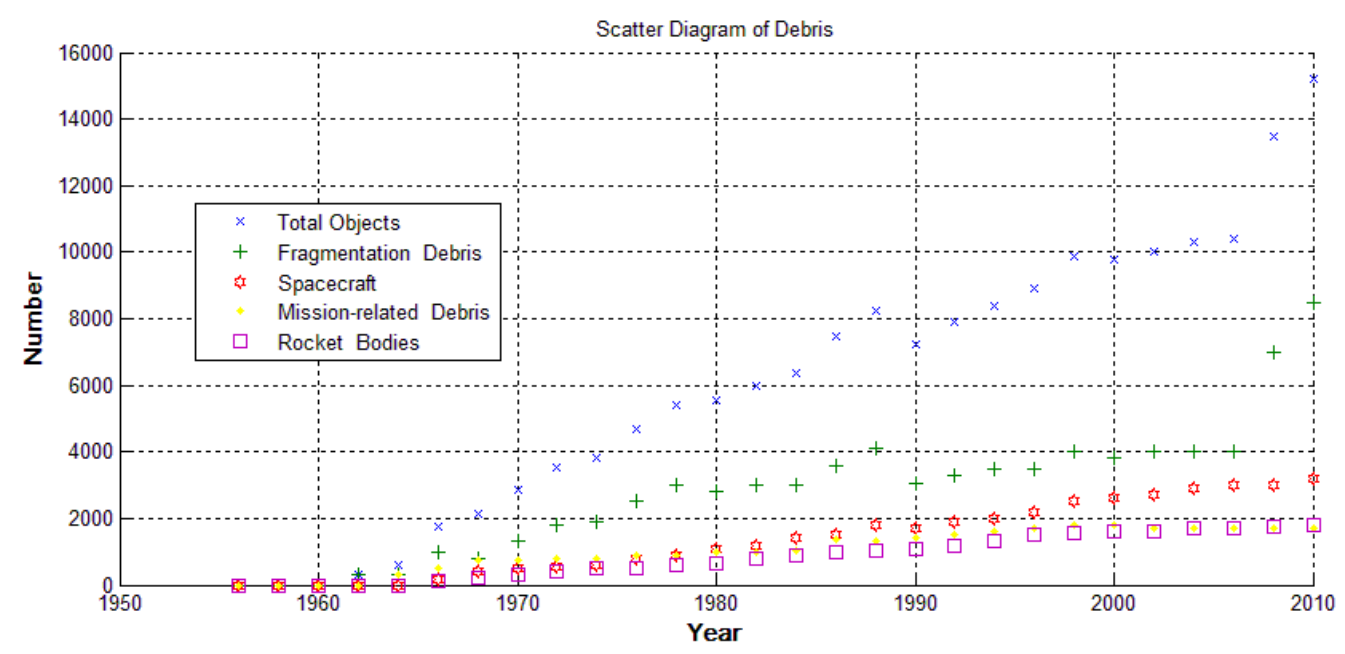

Figure .2Scatter Diagram of Debris

As Figure .2 shows, the purple scattered point shows a rising trend in the early stage, but in later term it tends to be stable. Through researching the literature, we know that more countries are taking active measures to lower their satellite's discarded problems which results in the mission-related debris decrease continuously. Therefore, we use its limit value of the scattered point distribution as its future prediction. For the red and yellow scattered point, they show the overall upward trend. For green scattered point, there are two large fluctuations caused by the collision between spacecraft. Meanwhile, Space debris is mostly caused by the collision among them, so we can consider the total number of space debris that is presented in blue and the green scattered point distribution show the same growth trend approximately.

Next, aimed to predict the growth trend of the space debris quantity, we apply the method of curve fitting to obtain the functions which can represent the growth trend. Considering the growth trend of various debris comprehensively, Quadratic Fit method is used to process the scattered point that are presented in blue, green and red in order to obtain the growth curve.

\section{Model Solutions}

Based on Figure2, we formulate a quadratic function $y=a x^{2}+b x+c$, applying the least square method to find the quadratic curve which makes the distance of scattered points the shortest.

Simulated Annealing algorithm to fit the non-linear curve process is to find the specific function $y$, so that the residual square and the minimum of the original data $y$, is a dynamic estimation of the parameters in the process. So we take the parameters of the function as aim to obtain specific values of the parameters by adopting the Maximum Likelihood Criterion. In the process of figuring out parameters, we adopt Simulated Annealing algorithm to obtain the best fitting performance, the specific workflow is as follows: 


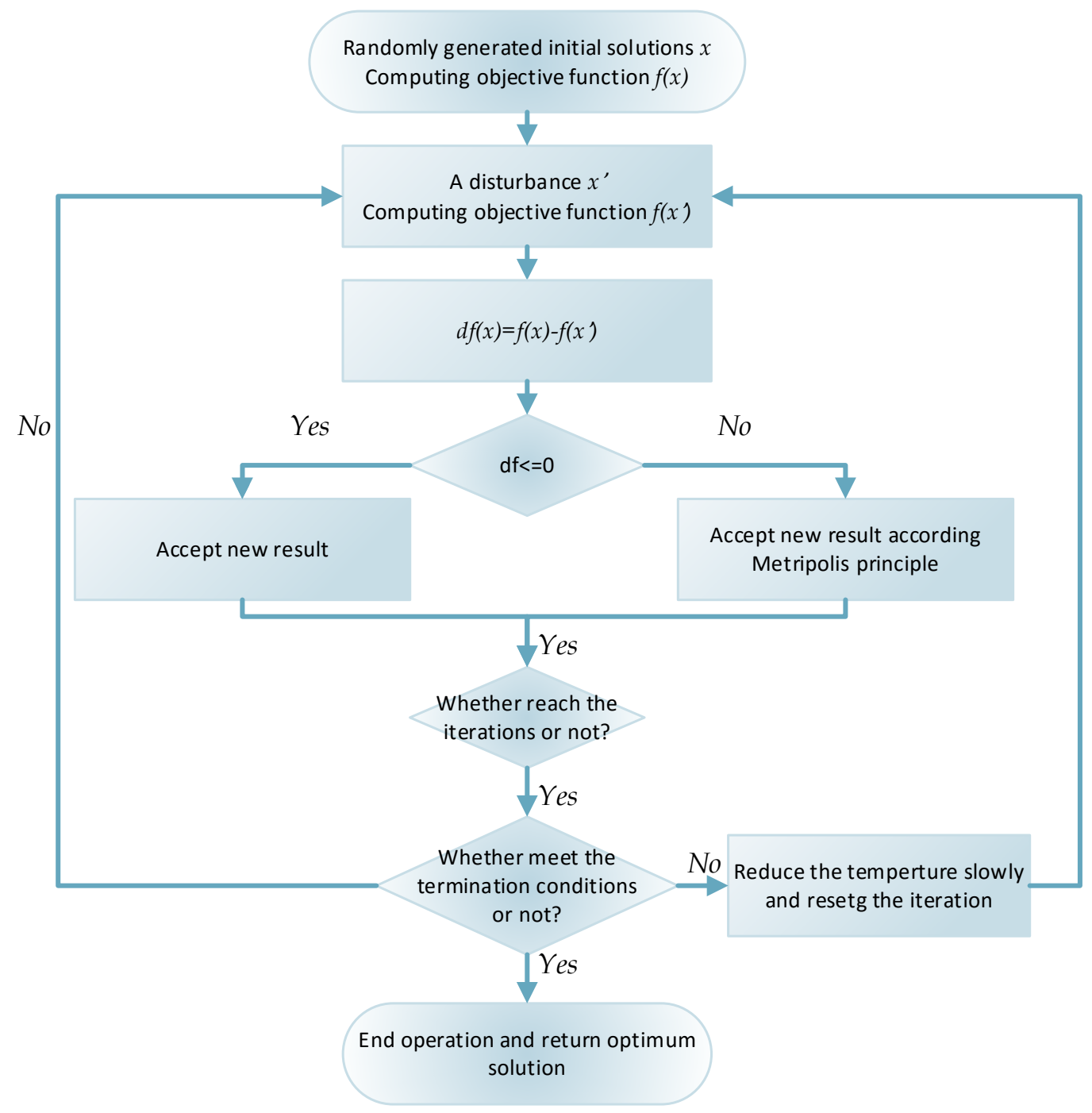

Figure 3.The specific workflow of Simulated Annealing algorithm

By applying the method of fitting to calculate with MATLAB, we obtain the growth trend of various types of space debris with time going by, and the formulations are shown as follows:

$$
\left\{\begin{array}{l}
Q_{\text {Total }}=1.472 t^{2}+459.2967 t-987.5186 \\
Q_{\text {Fra }}=1.6448 t^{2}+174.4339 t-194.8104 \\
Q_{\text {Spa }}=1.6154 t^{2}+84.0133 t-283.9498 \\
Q_{\text {Spa }}=1.6154 t^{2}+84.0133 t-283.9498 \\
Q_{\text {Mis }}=\left\{\begin{array}{l}
-2.0035 t^{2}+129.6994 t-267.5997(1950 \leq t<2040) \\
1560(t \geq 2040)
\end{array}\right. \\
Q_{\text {Roc }}=0.2472 t^{2}+70.1822 t-235.7015
\end{array}\right.
$$

Based on the formulations, we draw the growth curve of them with time going by and the specific result is as follows: 


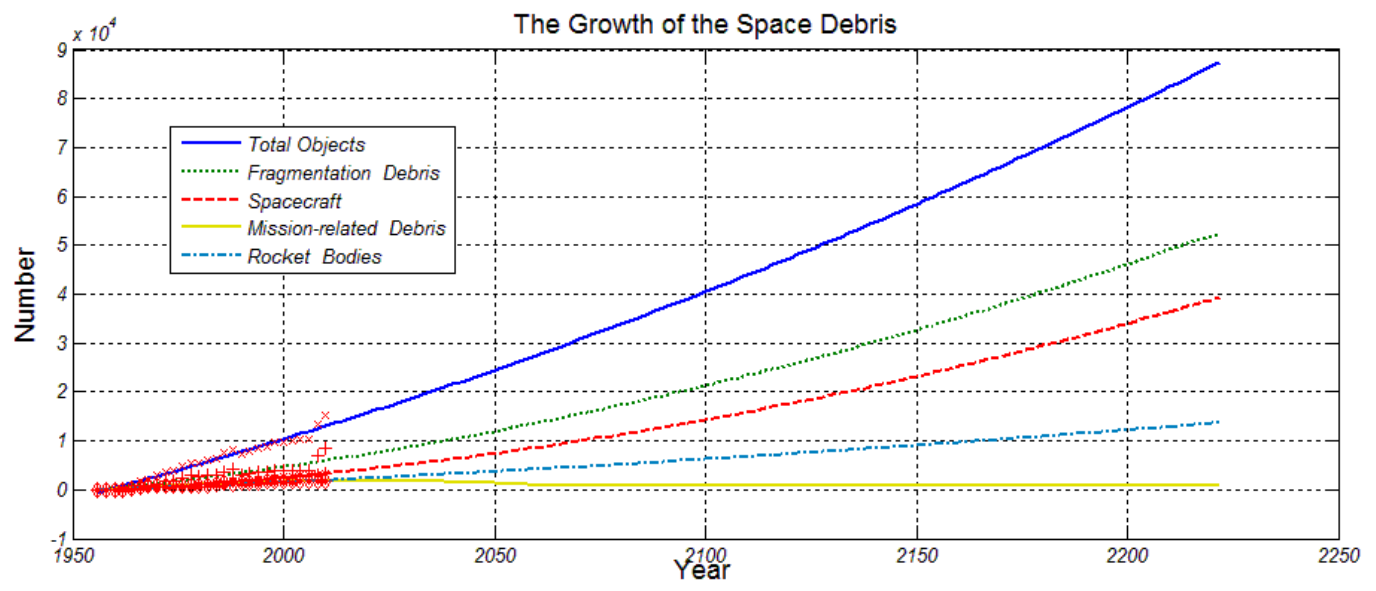

Figure .4The Space Debris Growth

From Figure .4, we can find that the total number of the space debris shows a continuous rise and the rising rate is also increasing continually since the probability of collision will increase with the growth of the space debris. We can conclude that our predication accords with the data given by NASA, which validate the accuracy and practicability of our model.

\section{Summary}

By the Space Debris Growth Model, we obtain the changing tendency of space debris quantity along with the time. It is shown that the quantity and rising rate continually increase.

\section{References}

[1] Hwang C R. Simulated annealing: theory and applications. Acta Applicandae Mathematicae, 1988, 12(1):108-111.

[2] Information on http://jyoder.com/

[3] Ansdell M. Active space debris removal: Needs, implications, and recommendations for today's geopolitical environment. Journal of Public and International Affairs, 2010, 21: 7-22.

[4] Chavent G. Identification of distributed parameter systems: about the output least square method, its implementation and identifiability Proc. 5th IFAC Symposium on Identification and System Parameter Estimation. 1979, 1:85-97

[5] Geourjon C, Deleage G.SOPM: a selfoptimized method for protein secondary structure prediction. Protein Engineering, 1994, 7(2): 157-164. 